\title{
loflupane II 23 injection (DaTscanTM) SPECT brain imaging in patients with movement disorders and/or dementia: preliminary report
}

Keywords: diagnostic accuracy, sensitivity, specificity, SPECT, ioflupane i123 injection, datscan, datscan, parkinson's disease, movement disorders, dementia

Abbreviations: AD, alzheimer's disease; DLB, dementia with lewy bodies; ET, essential tremor; MSA, multiple system atrophy; PD, parkinson's disease; PS, parkinsonian syndrome; PSP, progressive supranuclear palsy; SPECT, single-photon emission computed tomography; SWEDDs, scans without evidence of dopminergic deficit

\section{Introduction}

Early and accurate diagnosis of movement disorders and dementia is critically important to ensure patients receive appropriate treatment. Ioflupane I123 injection (ioflupane (123I) or (123I) FP-CIT or DaTscanTM or DaTscanTM) is approved in Europe and the US for diagnostic use only, for detecting loss of functional dopaminergic neuron terminals in the striatum in patients with clinically uncertain parkinsonian syndromes (PS), including those at an early stage, to help differentiate essential tremor (ET) from PS related to Parkinson's disease (PD), multiple system atrophy (MSA), and progressive supranuclear palsy (PSP) (Figure 1). Ioflupane (123I) is unable to discriminate between PD, MSA, and PSP. In Europe, Ioflupane (123I)

\author{
Volume I Issue I - 2014 \\ Igor D Grachev,' Zuzana Walker, ${ }^{2}$ Klaus \\ Tatsch, ${ }^{3}$ Eduardo Tolosa ${ }^{4}$ \\ 'Life Sciences, GE Healthcare, USA \\ ${ }^{2}$ Division of Psychiatry, University College London \& North \\ Essex Partnership University Foundation Trust, UK \\ ${ }^{3}$ Department of Nuclear Medicine, Municipal Hospital Karlsruhe, \\ Germany \\ ${ }^{4}$ Neurology Service, University of Barcelona, Spain
}

\begin{abstract}
Correspondence: Igor D Grachev, Medical Affairs, GE Healthcare- Life Sciences, 101 Carnegie Center, Princeton, New Jersey 08540, USA, Tel + I-609-5I 4-6857, Fax + |-609-5 |4-6040, Email grachevi@hotmail.com
\end{abstract}

Received: April 20, 2014 | Published: May 03, 2014

is also approving to help differentiate probable dementia with Lewy bodies (DLB) from Alzheimer's disease (AD). Ioflupane (123I) is not able to discriminate between dementia with Lewy bodies and Parkinson's disease dementia (Table 1).
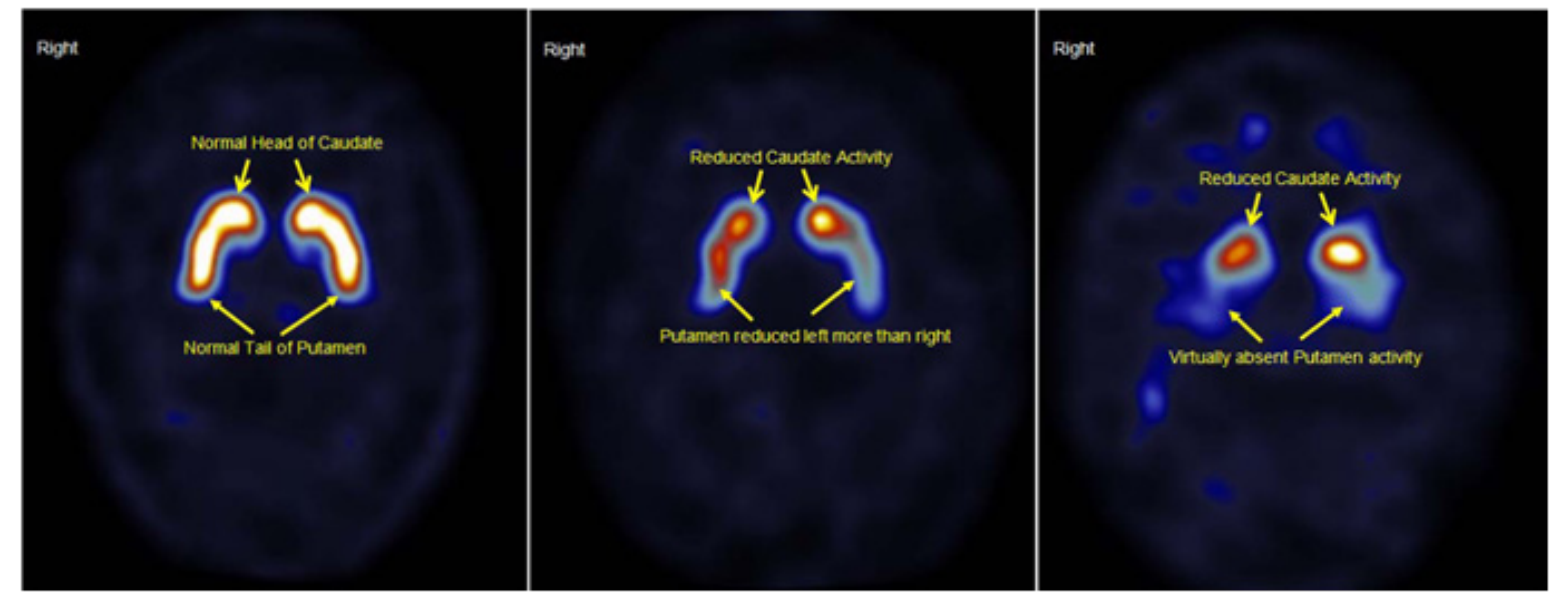

Figure I Example of Normal and Abnormal loflupane II 23 Injection SPECT Images.

The clinical differentiation of conditions with presynaptic dopaminergic deficit from those without such deficit is sometimes difficult. Diagnostic error, such as "over-calling" of PD, has been seen in autopsy studies, in which Lewy body degeneration was absent in vascular Parkinsonism. ${ }^{1}$ Several studies evaluating various medication protocols in patients diagnosed clinically as early PD had, in fact, normal imaging results in $4-14 \%$ of the cases..$^{2-4}$ In subjects with clinically uncertain PS, diagnoses were changed from PS to nonPS in $14.7 \%$ of subjects, as a result of normal imaging results, and changes to baseline diagnostic category are more likely to occur after
ioflupane(123I)imaging is performed than if it is not ( $45 \%$ vs. $9 \%$, respectively; $\mathrm{P}<0.001)^{5}$. In subjects with dementia with Lewy bodies, autopsy results revealed inaccuracies in early clinical diagnoses, particularly in discriminating from Alzheimer's disease. ${ }^{6,7}$

Extensive research has been conducted on the diagnostic effectiveness of Ioflupane (123I), but limited numbers of subjects were evaluated in each of the trials. We pooled four clinical trials ${ }^{8-13}$ to obtain a better estimate of the overall sensitivity and specificity of Ioflupane (123I) to detect or exclude a striatal dopaminergic deficit in 
subjects with a movement disorder or dementia. Within the 4 studies, 928 subjects were enrolled, 849 were dosed, and 764 completed their study. A total of 726 subjects were available for analysis in the intent-to-diagnose (ITD) population, and 622 in the per-protocol (PP) population. Images were classified as normal or abnormal by a panel

Table I loflupane II 23 Injection Approved Indications of 3 or 5 blinded experts and/or on-site nuclear medicine physicians. Figure 1 displays examples of normal and abnormal Ioflupane (123I) SPECT Images. These results were compared with expert clinical diagnosis, (obtained by a panel of experts in 2 of the 4 studies), ${ }^{9-11}$ the reference standard, to determine sensitivity and specificity.

\section{DaTscan $^{\mathrm{TM}}-$ US $^{18}$}

\section{DaTscan ${ }^{\mathrm{TM}}-\mathrm{EU}^{19}$}

This medicinal product is for diagnostic use only. DaTscan ${ }^{\mathrm{TM}}$ is indicated for detecting loss of functional dopaminergic neuron terminals in the striatum.

DaTscan (loflupane I I 23 Injection) is a radiopharmaceutical indicated for striatal dopamine transporter visualization using Single Photon Emission Computed Tomography (SPECT) brain imaging to assist in the evaluation of adult patients with suspected Parkinsonian Syndromes (PS).

In these patients, DaTscan ${ }^{\mathrm{TM}}$ may be used to help differentiate essential tremor from tremor due to PS (idiopathic Parkinson's disease, multiple system atrophy, and progressive supranuclear palsy). $\mathrm{DaTscan}^{\mathrm{TM}}$ is an adjunct to other diagnostic evaluations.
In adult patients with clinically uncertain Parkinsonian Syndromes, for example those with early symptoms, in order to help differentiate Essential Tremor from Parkinsonian Syndromes related to idiopathic Parkinson's Disease, Multiple System Atrophy and Progressive Supranuclear Palsy. DaTscan ${ }^{\mathrm{TM}}$ is unable to discriminate between Parkinson's Disease, Multiple System Atrophy and Progressive Supranuclear Palsy.

In adult patients, to help differentiate probable dementia with Lewy bodies from Alzheimer's disease. DaTscan ${ }^{\mathrm{TM}}$ is unable to discriminate between dementia with Lewy bodies and Parkinson's disease dementia.
The overall sensitivity was above $85 \%$ and specificity was above $90 \%$. Sensitivity and specificity were slightly lower in subjects with dementia with Lewy bodies than in subjects with parkinsonian syndrome. Across all studies, when images were assessed by on-site readers, Ioflupane (123I) imaging diagnostic effectiveness had an overall sensitivity of above $90 \%$ and specificity of above $80 \%$. This is a preliminary report, and study results will be published in full detail in the near future.

Despite obtaining high sensitivity and specificity overall, mismatches between the clinical diagnosis and dopamine transporter imaging results did occur. There are several possible explanations for why mismatches occur. One explanation is that during image acquisition, the level of dopamine transporter deficiency may be insufficient to show up on Ioflupane (123I) images. This is highly unlikely in PS, because dopamine cell loss has been shown to be greater than $50 \%$ at clinical presentation. ${ }^{14}$ It is also unlikely in DLB, because abnormal scans have been observed in patients in which Parkinsonism was minimal. ${ }^{10}$ Mismatches are more likely attributable to incomplete clinical features than imaging deficiencies. In PS, only one of three cardinal signs at presentation, two signs without bradykinesia, doubtful bradykinesia or postural rather than rest tremor might confound the diagnosis. ${ }^{5}$ Advanced PD cases with three cardinal signs including tremor syndrome predominance and responsive to L-Dopa typically diagnosed well without scan. Patients presenting with adult-onset dystonic tremor may mimic parkinsonian tremor and bradykinesia and have scans without evidence of dopminergic deficit (SWEDDs). ${ }^{15}$ Drug-induced Parkinsonism can mimic PD, including asymmetry of clinical features, and drug withdrawal is not always feasible to confirm the etiology. ${ }^{16}$ In DLB, clinical diagnostic confidence is low in cases of possible disease, with only approximately one quarter of them being confirmed pathologically as DLB. ${ }^{17}$ When Ioflupane (123I) imaging was performed in subjects with possible DLB, $38 \%$ showed abnormal results, ${ }^{10}$ supporting the helpfulness of a specificity value of $90.1 \%$.

This pooled analysis will demonstrate that the visual assessment of Ioflupane (123I) images provided high levels of sensitivity and specificity in detecting the presence or absence of a striatal dopaminergic deficit. In cases of diagnostic uncertainty, it is appropriate to use Ioflupane (123I) imaging when the diagnostic considerations include disorders that exhibit striatal dopaminergic deficit versus those that do not. Ioflupane (123I) brain imaging has the potential to improve diagnostic accuracy in patients with signs and symptoms of a movement disorder and/or dementia.

\section{Acknowledgments}

This pooled analysis was funded by GE Healthcare.

\section{Conflicts of interest}

a. IDG is an employee of GE Healthcare and does not hold any stocks or shares, or have other financial competing interests.

b. KT has received personal fees for performing DaTscan SPECT image reads during clinical trials and honoraria for lectures from GE Healthcare.

c. ET has received personal fees for research, lectures and consultancy from Novarits, TEVA, Boehringer Ingelheim, UCB, Solvay, and Lundbeck; grants from the Spaniard Network, Michael J. Fox Foundation, and Fondo de Investigaciones Sanitarias de la Seguridad Social. 


\section{References}

1. Hughes AJ, Daniel SE, Kilford L, et al. Accuracy of clinical diagnosis of idiopathic Parkinson's disease: a clinico-pathological study of 100 cases. J Neurol Neurosurg Psychiatry. 1992;55(3):181-184.

2. Schapira AH, Mc Dermott MP, Barone P, et al. Pramipexole in patients with early Parkinson's disease (PROUD): a randomised delayed-start trial. Lancet Neurol. 2013;12(8): 747-755.

3. Whone AL, Watts RL, Stoessl AJ, et al. Slower progression of Parkinson's disease with ropinirole versus levodopa: The REAL-PET study. Ann Neurol. 2003;54(1):93-101.

4. Fahn S, Oakes D, Shoulson I, et al. Levodopa and the progression of Parkinson's disease. N Engl J Med. 2004;351:2498-2508.

5. Kupsch AR, Bajaj N, Weiland F, et al. Impact of DaTscan SPECT imaging on clinical management, diagnosis, confidence of diagnosis, quality of life, health resource use and safety in patients with clinically uncertain parkinsonian syndromes: a prospective 1-year follow-up of an openlabel controlled study. J Neurol Neurosurg Psychiatry. 2012;83(6):620 628.

6. Litvan I, MacIntyre A, Goetz CG, et al. Accuracy of the clinical diagnoses of Lewy body disease, Parkinson disease, and dementia with Lewy bodies: a clinicopathologic study. Arch Neurol. 1998;55(7):969-978.

7. Walker Z, Jaros E, Walker RW, et al. Dementia with Lewy bodies: a comparison of clinical diagnosis, FP-CIT single photon emission computed tomography imaging and autopsy. J Neurol Neurosurg Psychiatry. 2007;78(11):1176-1181.

8. Benamer HTS, Patterson J, Grosset DG, et al. Accurate differentiation of parkinsonism and essential tremor using visual assessment of [123I]FP-CIT SPECT imaging: the [123I]-FP-CIT study group. Mov Disord. 2000;15(3):503-510.

9. Marshall VL, Reininger CB, Marquardt M, et al. Parkinson's disease is overdiagnosed clinically at baseline in diagnostically uncertain cases: a 3-year European multicenter study with repeat [123I]FP-CIT SPECT. Mov Disord. 2009;24(4):500-508.
10. Mc Keith I, O'Brien J, Walker Z, et al. Sensitivity and specificity of dopamine transporter imaging with 123I-FP-CIT SPECT in dementia with Lewy bodies: a phase III, multicentre study. Lancet Neurol. 2007;6(4):305-313.

11. O'Brien JT, McKeith IG, Walker Z, et al. Diagnostic accuracy of 123IFP-CIT SPECT in possible dementia with Lewy bodies. Br J Psychiatry. 2009;194(1):34-39.

12. Catafau AM, Tolosa E. Impact of dopamine transporter SPECT using 123I-Ioflupane on diagnosis and management of patients with clinically uncertain Parkinsonian syndromes. Mov Disord. 2004;19(10):1175-1182.

13. Tolosa E, Borght TV, Moreno E. Accuracy of DaTSCAN (123I-Ioflupane) SPECT in diagnosis of patients with clinically uncertain parkinsonism: 2 year follow-up of an open-label study. Mov Disord. 2007;22(16):23462351.

14. Fearnley JM, Lees AJ. Ageing and Parkinson's disease: substantia nigra regional selectivity. Brain. 1991;114(5):2283-2301.

15. Schneider SA, Edwards MJ, Mir P, et al. Patients with adult-onset dystonic tremor resembling parkinsonian tremor have scans without evidence of dopaminergic deficit (SWEDDs). Mov Disord. 2007;22(15):2210-2215.

16. Esper CD, Factor SA (2008) Failure of recognition of drug-induced parkinsonism in the elderly. Mov Disord. 23(3):401-404.

17. Verghese J, Crystal HA, Dickson DW, et al. Validity of clinical criteria for the diagnosis of dementia with Lewy bodies. Neurology. 1999;53(9):1974-1982.

18. http://us.datscan.com/wp-content/themes/main_site/pdf/prescribinginformation.pdf

19. http://www.ema.europa.eu/docs/en_GB/document_library/EPAR_Product_Information/human/000266/WC500035355.pdf 